\title{
REFLEXIONES METODOLÓGICAS SOBRE EL USO DE LAS HISTORIAS DE VIDA EN LA INVESTIGACIÓN DEL CAMBIO URBANO
}

John Harold Córdoba Aldana

\begin{abstract}
Resumen
El artículo que presento a continuación es una reflexión metodológica resultado de una investigación sobre el cambio urbano en Bogotá y el área metropolitana en los últimos 20 años. El trabajo de campo estuvo basado en un sistema de investigación conocido como método biográfico que incluyó información de tipo cuantitativo, representado en los censos de población de 1993 y 2005 y una encuesta aplicada en el área de estudio durante el año 2009. Así mismo, la metodología incluyó la elaboración de 66 historias de vida en la capital colombiana, en Barcelona (España) y en Paris (Francia). Este escrito es un análisis sobre la elaboración de estas historias de vida, que busca contribuir a la comprensión de este enfoque metodológico para futuras investigaciones en el campo de las ciencias sociales, particularmente en la geografia y que permitan dar cuenta de las transformaciones del espacio urbano.
\end{abstract}

Palabras clave: Historia de vida, urbanismo, investigación, Bogotá

\begin{abstract}
:
This paper is a methodological analysis as a result of Bogota's urban changing and metropolitan's area in the last twenty years. Fieldwork was based on a research system known as biographic method which includes quantitative information from 1993 and 2005 population census and survey applied within the research area in 2009. This methodology included 66 life histories from Bogotá (Colombia), Barcelona (Spain) and Paris (France). This paper is an analysis about creation of these life histories, which aims at contributing to the understanding of this methodological approaches for future researches in social science fields, particularly in Geography, so that allow to record urban space transformation.
\end{abstract}

Keywords: life history, urban. Research. Bogotá.

\section{Introducción}

El estudio de las transformaciones urbanas en la actualidad, recoge una larga tradición de investigaciones que hunde sus raíces en los primeros trabajos de la Escuela de Chicago en los Estados Unidos durante los años 20' (Grafmeyer, 2004 [1995]). Los cambios en las grandes ciudades han continuado de manera acelerada y sin cesar y no se detienen en el siglo actual. Bogotá, principal ciudad de
Colombia pasó en tan sólo un siglo de tener aproximadamente 100,000 habitantes para el censo de 1905 (Melo, 2001, p. 59) a 6,778,691 personas censadas en el 2005 (Dane, 2008, p. 29), sin contar con la población que habita el área metropolitana. Una pregunta que resulta para quien busque dar cuenta de tales cambios espacio-temporales es la forma de acercarse a este proceso y ofrecer una explicación comprensible. Existen múltiples entradas y en este artículo sólo voy a tratar una que busca recuperar la voz de los mismos 
habitantes de las ciudades, de sus historias, sus prácticas y representaciones urbanas a partir de la elaboración de historias de vida. En últimas, son los hombres quienes hacen la geografía y la historia.

\section{El contexto de la investigación}

El siguiente artículo es el resultado de una investigación sobrela movilidad internacional y el cambio urbano en la capital colombiana entre 1993 y 2009. La investigación de que se sirve este artículo, está inscrita en un contexto más amplio que articula el desarrollo de mi tesis doctoral en la Universidad de Rennes 2 en Francia, el proyecto ANR (Agencia Nacional para la Investigación - en Francia-) METAL (Metrópolis de América latina en la Mundialización), el apoyo de la Universidad Pedagógica Nacional en Colombia que me otorgó una comisión de estudios entre los años 2007-2010 y 2013 y el proyecto aprobado por la Facultad de Humanidades de la misma Universidad, durante el año 2012 para la elaboración de un análisis sobre el uso de las historias de vida en la investigación social. El propósito general es contribuir con una mirada crítica sobre las etapas recorridas en una investigación particular sobre la validez de este enfoque metodológico para dar cuenta de los diversos procesos espaciales.

La investigación en general, buscó establecer la relación entre dos procesos que muchas veces se observan de manera separada: la movilidad internacional y el cambio urbano, a través de las dinámicas residenciales. Bogotá no se caracteriza por una fuerte inmigración (en comparación con otros países latinoamericanos como Argentina, Chile o Brasil). Son $29.613^{1}$ habitantes, que según el censo de 2005 no habían nacido en Colombia y residían para la fecha en
Bogotá (Dane, s/f). Por otro lado, tiene gran importancia por su magnitud, la salida de población de la ciudad que corresponde a 55.159 hogares de la capital del país que tienen miembros en el extranjero ${ }^{2}$. Otras fuentes basadas en el mismo censo calculan que unos 617.307 bogotanos han salido de la ciudad a vivir fuera del país, es decir, el $9.11 \%$ del total de la población (KhoudourCastéras, 2007, p. 260). El conocimiento que existe sobre las idas y venidas (o retorno) de la población hacia y desde Bogotá, o incluso de antiguos migrantes que salieron de otras regiones del país y que regresan a la capital colombiana para fijar su residencia es casi desconocido. La información es insuficiente y muchas veces exagerada en el contexto de la crisis económica mundial. Sobre el tema está el trabajo del Grupo de Movilidad Humana quien revela que los bogotanos que regresaron a la ciudad para fijar de nuevo su residencia, provienen de Estados Unidos (43.3\%), Ecuador (15\%) y Venezuela (13.3 \%) (Mejía et al., 2009, p. 9). Para tratar estos complejos movimientos en el espacio, el enfoque metodológico tomó en cuenta los testimonios de algunos de sus protagonistas (individuos y familias), que permitieran un análisis sobre su papel en el cambio urbano.

\section{El método biográfico y la elaboración de las historias de vida}

El enfoque biográfico ha sido abordado como metodología en distintas investigaciones sobre la migración y la movilidad espacial (Mejía et al., 2009, p. 6). Este método da luces sobre las distintas formas de la movilidad en lugar de centrarse en la historia residencial de un individuo en un momento dado. Hace énfasis en los procesos socio-espaciales cuya historia se reconstituye durante el trabajo de campo.

1 Inmigrantes internacionales de toda la vida. No incluye la localidad 20 (Sumapaz) de Bogotá, ni la población rural. Con Sumapaz y la población rural son 30.395 habitantes.

2 La información del Censo de 2005 sólo permite obtener datos a partir de los informantes en Bogotá. No hay datos sobre los hogares completos que salieron de la capital o de antiguos emigrantes que no tienen hogares que los refieran. 
L'originalité de l'approche biographique réside dans le développement de nouvelles méthodes statistiques et la réalisation d'enquêtes permettant de les tester. [...] Ces enquêtes permettent de resituer la mobilité para rapport aux événements qui jalonnent l'existence des individus. L'apparition de nouvelles méthodes mais aussi les progrès de l'informatique ont permis de dépasser l'approche purement individuelle en réalisant le passage de l'histoire d'un individu à celle d'un échantillon d'individus. Elles ont également permis le passage de l'analyse d'un parcours résidentiel à celle d'une biographie c'est-à-dire de plusieurs parcours en interaction : parcours résidentiel, familial, professionnel (Bonvalet y Brun, 2002, p. 36).

Una de las herramientas que se utiliza en el método biográfico es la elaboración de historias de vida que permiten reconstruir las trayectorias residenciales individuales y colectivas. Las historias contadas por los individuos son diferentes de unos a otros $\mathrm{y}$ es precisamente esas particularidades, sin olvidar las condiciones estructurantes, las que son exploradas a partir de esta metodología. Más que un recorrido sobre la historia del cambio de vivienda de un individuo, la trayectoria residencial merece toda la atención para la comprensión e interpretación de las recomposiciones del espacio urbano.

A grandes rasgos, el método propuesto continúa una larga tradición metodológica de la que se encuentran distintas denominaciones como relato de vida, historia de vida ${ }^{3}$, entrevistas en profundidad, enfoque biográfico, autobiografía, etc. Esta metodología ha tomado fuerza dentro de las ciencias sociales, por lo menos desde la década de los 70'. Para Bertaux (1980), en el periodo de entreguerras fueron conocidas las elaboraciones de historias de vida y relatos de vida de la escuela de Chicago o el trabajo sobre la memoria de los obreros en Polonia. Sin embargo, luego de la Segunda Guerra Mundial, su uso comenzó a ser marginal hasta que distintas formas de conocimiento social, entre ellas la sociología, la antropología, la sicología o la historia comienzan a recuperarla, con diferente intensidad, unas décadas después.

Buena parte de las investigaciones que incorporan la elaboración de historias de vida, particularmente desde la disciplina histórica, se han elaborado sobre la Segunda Guerra Mundial, los campos de concentración y los testimonios de los prisioneros de guerra. Estas investigaciones tienen como particularidad recuperar la memoria histórica de las personas que participaron en estos hechos y que se han trasladado a temáticas como la Guerra de Vietnam, la bomba atómica sobre Hiroshima o el Holocausto con el propósito de lograr procesos de reconciliación (Andrews et al., 2006, p. 156).

Pero el uso de la historia de vida no es sólo un enfoque de la disciplina histórica. También ha demostrado su potencialidad en la psicología por sus características peculiares que permiten establecer el vínculo entre el individuo y la sociedad (Ramos y Romero, 2006, p. 23). Su uso se da porque incluso hablar del pasado de un individuo puede tener un valor terapéutico frente a hechos que pudieron resultar en ocasiones dolorosos (pérdida de la vivienda o la pareja) o la poca posibilidad que tiene los sujetos de hablar sobre sus vidas y entrar en contacto con otras personas.

3 Bertaux (1980) diferencia el relato de vida de la historia de vida. El primero de los términos designa la historia de una vida tal y como la cuenta el que la vivió y el segundo se reserva a los estudios de caso sobre una determinada persona incluyendo, además del propio relato, otro tipo de documentos. El método biográfico corresponde a un amplio proceso de investigación donde se recolectan relatos de vida que continuamente son cuestionados según los propósitos de la investigación. La historia de vida autobiográfica corresponde a la construcción de una narrativa construida a partir de la historia de un solo individuo. 
Pero no hay que confundir la elaboración de historias de vida con el método biográfico que las incluye, así como la aplicación de encuestas que también hacen un recorrido en la historia residencial de los individuos. Por ejemplo, la elaboración de encuestas biográficas para dar cuenta de los procesos migratorios y profesionales, ya completa por lo menos treinta años de uso en los estudios demográficos, y su utilización se ha hecho extensiva a la geografía. Las investigaciones que recurren al método biográfico en el medio francés son múltiples. Lelievre anota las características de la encuesta biográfica realizada en la India entre 1987 y 1991, orientadas por Dupont, donde no sólo hace parte el empleo de las trayectorias residenciales sino que se amplía a una lectura longitudinal sobre la carrera profesional y el ciclo de vida de la familia con el propósito de establecer la interacción entre los distintos elementos (Lelièvre, 1999, p. 197).

En Colombia la elaboración de historias de vida en las ciencias sociales se remonta por lo menos 30 años atrás. En 1992 el Centro de Investigaciones sobre Dinámica Social (CIDS) de la Universidad Externado de Colombia organizó el seminario internacional El uso de las historias de vida en ciencias sociales: teorías, metodologías y prácticas en la ciudad de Villa de Leyva. Como resultado fueron publicados dos tomos que recogen la mayor parte de las ponencias presentadas en dicho evento. Luego, el número de investigaciones fueron creciendo en la medida en que la década de los 90' avanzaba. Londoño (1998) elabora una biografía de Juan de la Cruz Varela en la que combina distintas fuentes para dar cuenta de la historia social y las luchas agrarias de la región del Sumapaz (Colombia). Así mismo, diversas investigaciones fueron presentadas en los encuentros nacionales de historia oral que se llevaron a cabo en Bogotá (Colombia) durante los años 2005 y 2010 liderados por el Colectivo de Historia Oral (http:// colectivohistoriaoral.wordpress. com). También han sido importantes las publicaciones sobre la enseñanza de la historia, que hacen uso de la historia oral, realizadas en el país, de las cuales se sirvió como referente la investigación que presento en este artículo (Castro, 2004; Vega y Castaño, 1997).

Para mi investigación, las entrevistas no buscaban abarcar la totalidad de la historia de vida de los entrevistados. En ese sentido, la estructura se acerca más al concepto de historia de vida temática como lo definen Riley y Harvey (2007, p. 393), para referirse al énfasis sobre un aspecto en particular de la historia de vida de la persona, en su caso particular el tema de la agricultura y el cambio del paisaje rural. En este caso en particular, sobre la movilidad internacional y las transformaciones urbanas en la ciudad de Bogotá.

A partir de los antecedentes que tenemos sobre el uso de la historia de vida, se pueden anotar tres tipos de aportes en mi estudio y que a la vez se convierten en una contribución metodológica para la geografía (Bertaux, 1980; Cailly, 2007, pp. 171-172; Riley y Harvey, 2007). En primer lugar, la relación del enfoque investigativo con las ciencias sociales. En segundo lugar, los aportes metodológicos sobre el tema en particular, es decir, el uso de las historias de vida para dar cuenta de una de las dimensiones de la movilidad internacional y las dinámicas residenciales vinculadas con las prácticas urbanas, las representaciones y la escogencia residencial que de allí derivan. En tercer lugar, sobre los aspectos metodológicos en la elaboración de las entrevistas. Estos aspectos son desarrollados a continuación.

\section{Múltiples entradas al estudio de las transformaciones urbanas.}

El método utilizado se definió como un sistema de investigación con varias entradas, que no privilegió sólo un enfoque para analizar la relación entre la movilidad internacional y las dinámicas residenciales 
en Bogotá. En la figura 1 aparece en la parte superior las múltiples escalas que permitieron abordar el tema de la movilidad internacional y cada una de las fuentes que integraron el método biográfico.

Figura 1. Sistema de investigación

\section{Autor: John Harold Córdoba Aldana}

Una de las características de la investigación es que el análisis se desarrolló a partir de tres escalas. Una que podemos llamar metropolitana que incluye la capital colombiana y algunos municipios que están en su área de influencia; otra zonal que corresponde a las 12 zonas seleccionadas dentro del proyecto METAL y donde se aplicó la encuesta de movilidad espacial 2009. Por último, una más local que corresponde a tres barrios de Bogotá y un municipio de la metrópoli bogotana. Estas últimas zonas o barrios podrían ser ilustrativos de los cambios que se vienen dando a nivel metropolitano, teniendo en cuenta las relaciones multiescalares.

\section{Las historias de vida en la escala local}

La delimitación del Área Metropolitana de Bogotá no corresponde a una definición administrativa debido a que tal organización del territorio no tiene un reconocimiento jurídico. Sin embargo, varios estudios han señalado un área funcional compuesta por 19 localidades de Bogotá y 19 municipios: Bojacá, Cajicá, Chía, Cota, El Rosal, Facatativá, Funza, Gachancipá, La Calera, Madrid, Mosquera, Sibaté, Soacha, Sopó, Subachoque, Tabio, Tenjo, Tocancipá y Zipaquirá (Dureau et al., 2011, p. 20). En segundo lugar están 12 zonas dentro del área metropolitana en la que se aplicó una encuesta a 881 hogares: La perseverancia, La Candelaria, El Nogal, Normandía, Gustavo Restrepo, San Cristóbal Norte, Bosa, Chía, Madrid, Soacha, Ciudad Salitre y Calle 80 que se pueden observar en la figura 2. Por último, el proyecto profundiza en las prácticas y representaciones sociales en cuatro zonas de la ciudad donde existe presencia de distintas formas de movilidad internacional: La Candelaria, La Perseverancia, El Nogal y Chía. En la escala local es donde las historias de vida fueron más útiles durante la investigación.

Igualmente, fueron elaboradas historias de vida en dos ciudades europeas. La escogencia de Barcelona y Paris como ciudades de estudio, obedeció a que estas ciudades cuentan con un número importante de migrantes provenientes de los tres países latinoamericanos. Para el caso colombiano, la Comunidad Autónoma de Cataluña es el segundo lugar de destino después de Madrid en el periodo que va de 1998 a 2006 (Dureau, Flórez, y Hoyos, 1994, p. 74). El informe estadístico presentado por el Ayuntamiento de Barcelona en el 2011, da cuenta de la población extranjera en esta ciudad en el periodo 2002-2011. Los colombianos en esa ciudad eran para el 2011 12,612 habitantes. Ocupaban el octavo lugar entre la población extranjera (liderado por Pakistán) y el cuarto país latinoamericano (en los tres primeros lugares está Ecuador, Bolivia y Perú) (Ajuntament de Barcelona, 2011, p. 12). 
Figura 2. Delimitación de las zonas de estudio en Bogotá

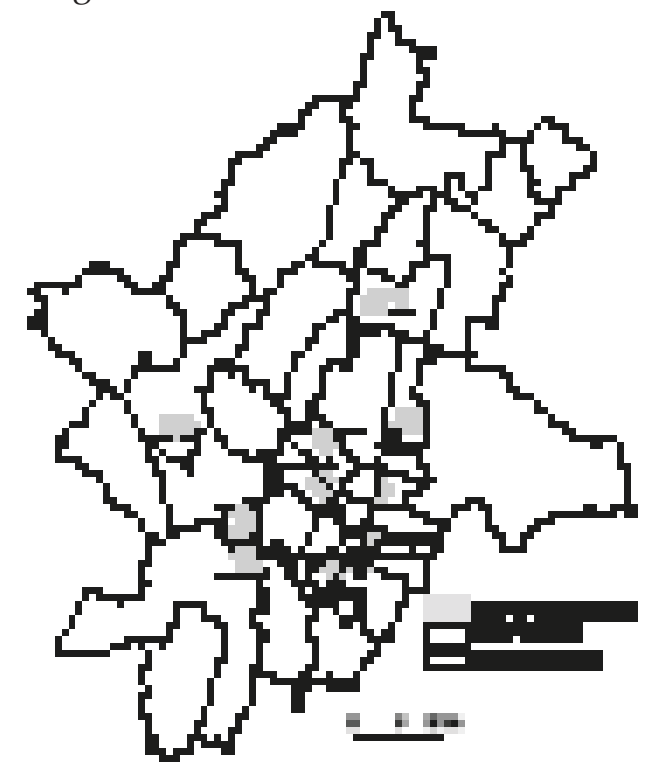

Fuente: Mapa elaborado por Le Roux (Dureau et al., 2011, p. 22).

\section{La elaboración de las historias de vida}

A continuación se describen cada una de las etapas que se tuvieron en cuenta al momento de realizar las historias de vida. En el trabajo de campo no se presentaron etapas sucesivas, por el contrario ellas se superponían con el propósito de no perder los contactos por la alta movilidad que en la tres ciudades se podía presentar. Para desarrollar esta etapa se crearon guías de entrevista y fichas para sistematizar la información que se obtenía de primera mano.

La guía para las entrevistas en Bogotá está diseñada de acuerdo a la estructura que se muestra en el recuadro 1. El instrumento desarrollaba tres aspectos generales. El primero, estaba enfocado en los cambios residenciales durante la historia de vida del entrevistado. El segundo, en los espacios de vida actual del hogar en diferentes escalas. El tercero en las distintas representaciones urbanas del entrevistado en torno a temas como la segregación, topofobias, topofilias, inseguridad, globalización y las que surgieran en el dialogo.
Recuadro 1. Estructura de la guía de entrevistas en Bogotá

\section{La guía de entrevista está estructurada en tres módulos.}

En el primer módulo, Historia de vida, se trata de entender el por qué de los cambios y las estrategias de las personas y de retomar la historia de vida (ya recolectada por medio de la encuesta por cuestionario) en relación con el contexto familiar y el contexto del barrio. Al conocer los datos recolectados en el cuestionario, se pueden hacer hipótesis sobre las articulaciones y las superposiciones de las etapas de la historia de vida y tratar de comprobarlas durante la entrevista.

El objetivo del segundo módulo, Espacios de vida del hogar en el presente, es explorar la distribución espacial y las prácticas de las actividades de los miembros del hogar a distintas escalas: las cotidianas, semanales, habituales o excepcionales en relación a la educación, el trabajo, el consumo, el ocio, la religión, etc. Se tratar de conocer: i) los territorios y micro-territorios locales, es decir lo que se puede llamar el espacio de vida, alrededor de la vivienda y en el barrio; ii) las redes en la ciudad, o sea los lugares conocidos donde se desplaza pero sin tener una relación fuerte con el entorno espacial. Se consideran dos formas distintas y complementarias del espacio geográfico, el territorio o micro-territorio y la red, que corresponden a dos escalas geográficas: la escala local (prácticas en el barrio alrededor de la vivienda) y la escala de la ciudad (prácticas en toda la ciudad con medios de transporte diferente y tiempos de desplazamientos más importantes).

En el tercer módulo, Representaciones locales y urbanas, se propone realizar un ejercicio de "cartografía o mapa mental" al entrevistado pidiéndole hacer un mapa de los lugares considerados como representativos de Bogotá. El ejercicio tiene como propósito hacer visible desde esta herramienta, la percepción sobre el espacio de Bogotá a varias escalas: el micro territorio de la vivienda, el barrio y la ciudad. El objetivo es identificar las representaciones estructurales, y captar lo que piensan las personas entrevistadas y cómo se representan en el espacio. Importa tanto lo que se dibuja como lo que se dice mientras se está dibujando o después.

Fuente: (Dureau, et al., 2011, p. 17) 
En el caso de la guía elaborada para las entrevistas en Barcelona y Paris,

“...se buscó captar la trayectoria migratoria, el papel de la estadía en Europa en estas trayectorias para los individuos y sus familias, las modificaciones de las prácticas urbanas cotidianas o de los modelos residenciales familiares en relación con la migración internacional, así como las relaciones con los miembros de la familia presentes en la ciudad de origen. También indagó sobre las transferencias directas o indirectas de estas personas hacia la ciudad de origen, en términos de compra o alquiler de vivienda y de las posibles transformaciones en las prácticas espaciales de los miembros de la familia que se quedaron o cuando los entrevistados regresan durante breves periodos de tiempo. Los cambios en los usos y representaciones espaciales de los migrantes en las ciudades europeas, pueden ejercer transformaciones territoriales en las ciudades de origen." (Dureau, et al., 2011, p. 11)

Así mismo, la guía de entrevista desarrollaba cuatro aspectos. El primero recolectaba la historia de vida de los individuos centrados en sus trayectorias migratorias, residenciales, profesionales y familiares; el segundo sobre las prácticas urbanas en las distintas trayectorias en la ciudad de origen y en las ciudades europeas; el tercero sobre las relaciones con el lugar de origen; y cuarto los proyectos personales. De ese modo se lograba una estructura general que permitía lograr los propósitos de la investigación y reconocía el papel central del entrevistado desde su propia experiencia de vida.

\section{Bogotá. Las etapas del trabajo de campo}

El trabajo de campo se llevó a cabo durante el segundo semestre de 2009 y tuvo como punto de partida las 881 encuestas realizadas en el marco del proyecto METAL de las cuales resultaron 136 con experiencia migratoria internacional. La revisión de los formularios arrojó 62 individuos encuestados $\left(\mathrm{ego}^{4}\right)$ con experiencia migratoria internacional, 46 individuos fuera de ego también con experiencia migratoria internacional y 130 individuos familiares de ego nacieron o vivieron fuera de Colombia. Al final, 136 formularios de encuesta tienen algún tipo de experiencia migratoria internacional.

La escogencia de los potenciales entrevistados se llevó a cabo durante el mismo proceso de elaboración de las encuestas. En compañía del investigador Hernando Sáenz íbamos seleccionando los formularios y luego las personas eran contactadas por teléfono para solicitar una cita, con el propósito de profundizar en algunos aspectos de la encuesta. Como la prioridad era entrevistar personas con experiencia migratoria internacional, cuando las personas no respondían al teléfono, iba directamente a las viviendas para solicitar las citas. En tres ocasiones que se presentó esta situación, dos fueron exitosas y en la tercera, no respondieron en la vivienda. Hay que agregar que este trabajo de campo en algunos casos, que no fueron la mayoría, porque ya existía la mediación de la encuestadora, había un poco de desconfianza. Por ejemplo, hubo varios intentos por teléfono y visita a la vivienda para entrevistar a una mujer que tenía un hijo en al ciudad de Barcelona, y sólo se pudo obtener una vez su familiar fue contactado en la capital catalana. El hecho de que su hijo fuera entrevistado fuera del país era una prenda de garantía en Colombia. 
El propósito inicial era realizar entrevistas al $10 \%$ de los hogares que resultaron de la encuesta cuantitativa, tomando como criterio de escogencia aspectos relevantes para el grupo de investigación en cada una de las zonas de la ciudad. En el caso de los formularios seleccionados para tratar el tema de la migración internacional en Bogotá, quien escribe este artículo, fue el responsable de tomar contacto y elaborar las entrevistas para las historias de vida.

A partir de la selección de los formularios, un total de 36 entrevistas (32 en una primera etapa, complementadas con 4 luego del trabajo de campo en las dos ciudades europeas $^{5}$ ) fueron realizadas en Bogotá y el área metropolitana a personas o familias con algún vínculo con la migración internacional. Así mismo, el trabajo de campo, sobre todo la elaboración de las historias de vida y el contacto directo con las personas, indujo a una reflexión de orden metodológico que permitió establecer relación con las ciencias sociales en general, de la cual emergieron los siguientes aspectos que quiero compartir:

- Los relatos de vida no son la verdad en sí misma y son incompletos. Son una fuente de información que tienen la misma naturaleza de otros documentos (información de prensa, documentos institucionales, fotografías, etc.). Son una expresión parcial e incompleta de la trayectoria, de la personalidad y la mentalidad de un individuo. No son una versión terminada o totalmente válida sobre los hechos que alude. En ese sentido, el enfoque biográfico debe combatir el fetiche de la biografía. "[...] la segmentación que opera en la realidad misma debe reconocerse como tal y ser incluida en el principio de la recolección de los relatos de vida" (Bertaux, 1980). Bertaux señala que cada vez más los individuos han sido arrojados a llevar vidas paralelas que pasan por el trabajo, la familia y el compromiso personal. En ese sentido, los relatos de vida no son la realidad misma, son una representación de la misma. Las dos dimensiones no son equivalentes.

- El insumo de la elaboración de la historia de vida es la memoria, el recuerdo del entrevistado, que es selectiva, reiterativa y reinterpreta la vida desde el presente. Las formas de valoración o las prácticas $\mathrm{y}$ representaciones que surgen en la investigación son resultados del presente. Es probable que la perspectiva que se tiene ahora sobre la historia de vida, el entrevistado no la tuviera en el pasado cuando la vivía. Toma en cuenta los sentidos que las personas le otorgan al pasado, acorde con sus experiencias y al desarrollo de su propia existencia (Grafmeyer, 2004 [1995], p. 23).

- Las historias orales al estar incrustadas en el presente son interpretaciones del pasado. Ellas permiten alimentar el debate sobre las transformaciones del paisaje y los cambios en las prácticas culturales (Riley y Harvey, 2007, p. 392). La historia oral permite comprender el cambio del paisaje, el lugar o el territorio desde sus protagonistas. En nuestro caso, sobre los cambios urbanos.

- Los relatos de vida no son sólo crónicas de los acontecimientos vividos, son además esfuerzos por darle sentido al pasado desde la situación presente y contienen en sí mismo los proyectos futuros.

- Importa tanto el recuerdo como el olvido, lo que se suprime, lo que se da por implícito, lo que se da por sentado y a las expresiones espontáneas que podrían pasar como desapercibidas durante las entrevistas.

- Permiten determinar la comprensión que tienen los entrevistados sobre los cambios actuales y su participación en el ordenamiento futuro de la ciudad.

5 Las 4 entrevista complementarias fueron realizadas el mes de marzo de 2010. 
- Las Ciencias Sociales han atribuido la capacidad que tienen las historias de vida para rescatar las voces subalternas y de las culturas ágrafas. Sin embargo, también contribuye al análisis de diversos aspectos personales y colectivos de diferentes grupos sociales.

- Los testimonios de las personas se encuentran situados no solamente en historias locales y en la creación de lugares sino también en la historia de lugares. Las narrativas expresan dimensiones sociales y culturales que con otros enfoques metodológicos podrían quedar por fuera. A través de las historias de vida se pueden tratar cuestiones de representación histórica de la vida social, lugar y paisajes (Andrews, et al., 2006, p. 177).

Sobre el tema de la movilidad internacional y el cambio urbano en Bogotá, quedan como reflexiones:

- Esta perspectiva permite analizar los modos de vida, las actitudes, representaciones y valores de conjuntos de población que pueden, en este caso, conducir a las escogencias residenciales a partir de las experiencias de vida.

- La entrada a partir de la historia de vida de las personas entrevistadas busca contribuir a la comprensión de las escogencias residenciales en relación con las prácticas y representaciones urbanas que en una perspectiva mucho mas amplia, es una contribución a la geografía urbana.

- Reconstruye el sentido de la escogencia residencial a partir de la palabra y las representaciones individuales. Considera la integralidad de todos los valores espaciales movilizados por un individuo, en tanto fuente de caracterización de las escogencias residenciales, sea cual sea su finalidad. La escogencia residencial al estar relacionada con otros procesos, a través de la historia de vida se puede acercar a su integralidad. López (1998, p. 89) establece este vínculo con la movilidad social en Venezuela a partir de la elaboración de historias familiares en tres generaciones en los que involucra los niveles de instrucción, trayectorias profesionales, espacios habitados, modos de vida y cambios en los elementos simbólicos.

A continuación quiero señalar algunos aspectos de orden metodológico, que por más insignificantes que parezcan, hacen parte del proceso de formación como investigador social. El olvido de algunos pequeños detalles, como me sucedió en algunas ocasiones, puede echar al traste una grabación o que no haya una buena comunicación con el entrevistado. Antes y durante se debe considera lo siguiente:

- Aunque parezca trivial, la puntualidad (llegar unos minutos antes de la hora programada), la amabilidad (saludar y despedirse de manera cordial), la postura frente al entrevistado, la claridad en las preguntas, que deben ser etnográficas, es decir, intentar adecuarse al lenguaje del entrevistado, son importantes para un buen desarrollo al dialogo y permitir otras sesiones o la recomendación con personas que pudieran ser entrevistadas.

- Igualmente, tener preparada la grabadora y conocer correctamente su funcionamiento. Colocarla en el lugar indicado (un entorno cerrado) y tener baterías de repuesto nos pueden ahorrar muchos problemas en el momento de la transcripción. Las entrevistas en espacios abiertos, donde haya paso continuo de personas (parques o cafeterías), no son la mejor idea. En mi caso, al inicio del trabajo de campo y sin mucha experiencia con mi equipo nuevo, perdí varios minutos de una entrevista porque no había prendido la grabadora y en otra ocasión porque las pilas se descargaron durante el dialogo. Por fortuna sólo fueron pocos, pero valiosos minutos. 
- Siempre hay que tomar fotos ya sea del entorno de la vivienda y ojala de la vivienda misma. Es una memoria que nos activará algunos aspectos que pueden ser relevantes en el momento del análisis.

- El entrevistado es la persona en quien reposa el conocimiento. Es decir, el informante está mejor informado que el entrevistador sobre su propia vida y la experiencia que tiene entorno al tema que se estudia. De esa manera se rompe con el monopolio del conocimiento en el cual puede reposar el poder de la ciencia. Hay que hacérselo saber o sentir a nuestro entrevistado. Hay que escuchar más de lo que se habla.

- Con el paso del tiempo me di cuenta que el relato que se cuenta, no es natural, es inducido o auto inducido. Por tanto, es necesario provocar una conciencia reflexiva en el entrevistado sobre su propia vida, para que ponga en perspectiva el recuerdo sobre el pasado. Por ejemplo, si los criterios de escogencia de la vivienda están ajustados a los valores que se manejaban en el pasado y su relato no refleja los del presente.

- El entrevistador no sólo escucha. También cuestiona, hace énfasis, busca otros caminos para nutrir las respuestas o verlas desde otro punto de vista.

- Por último, el dialogo con el entrevistado supera los mismos propósitos de la investigación. Se navega por aguas diferentes a las científicas. Es muy usual que en algunos pasajes los entrevistados lloren, rían, tengan rabia, suspiren, etc. Hablar del pasado, ejercicio que no es muy común activa múltiples emociones que deben ser manejadas de manera adecuada. A veces es mejor guardar silencio, alcanzar un pañuelo y orientar el dialogo a los objetivos propuestos. Hacer una historia de vida, sin duda, tiene un valor terapéutico que no es esperado antes del trabajo de campo. En muchos casos queda una relación extraña con el entrevistado, desde luego han contado su vida a otro extraño y con un café se espera volver a encontrar el momento para volver a hablar de la vida.

\section{Las historias de vida de los bogotanos en Europa}

Durante la encuesta y ya elaboradas las primeras entrevistas en Bogotá, con el objetivo de identificar el papel directo o indirecto en las transformaciones en la ciudades de origen, fueron seleccionados un subconjunto de formularios que tuvieran alguna relación con la migración en Barcelona o Paris (la última ciudad como segunda opción). La idea era poder relacionar los resultados del trabajo de campo en las ciudades europeas con los otros resultados de METAL en la metrópolis bogotana.

El formulario de la encuesta en su diseño permitía anotar la información sobre alguna forma de contacto de personas vinculadas con el hogar, que para ese momento pudieran estar residiendo en las dos ciudades europeas. Con el fin de realizar las entrevistas en Barcelona se llevaron a cabo dos misiones en el contexto del programa METAL. La primera del 17 al 30 de Junio de 2009 por parte de dos investigadoras asociadas al proyecto ${ }^{6}$, al mismo tiempo que se desarrollaba la encuesta en Bogotá. La segunda misión se llevó a cabo por mi, del 15 al 28 de Octubre del mismo año. Un total de 23 entrevistas fueron elaboradas durante las dos misiones.

En la ciudad de Paris, que estaba como segunda opción hasta completar 30 entrevistas, también se llevaron a cabo dos misiones. La primera del 24 al 30 de junio de 2009 donde Michelle Guillon, investigadora del programa, junto a las entrevistas que realizaba con inmigrantes de Santiago (Chile), elaboró dos historias de vida de

6 Naik MIRET y Michelle GUILLON. 
bogotanos que habían sido referenciados en la encuesta en Colombia. La segunda misión realizada por mí, se llevó a cabo del 10 de Noviembre al 7 de Diciembre de 2009. Un total de 7 entrevistas fueron realizadas durante las dos misiones.

La prioridad, puesto que la investigación estaba muy avanzada en Bogotá y que ya había un cierto número de contactos, fue el método definido como "relación con ego" (contactar los miembros de la familia o de los amigos de las personas encuestadas en Bogotá). En la medida en que se avanzaba en la encuesta de Bogotá, el equipo de trabajo enviaba la lista con los contactos de familiares o amigos en Barcelona y Paris, para tomar el contacto en las ciudades europeas. Esta primera etapa fue larga y difícil, y rápidamente se vio que no encontraríamos suficientes contactos en esta muestra; se optó por una segunda muestra de tipo bola de nieve, a partir de personas de las redes relacionales con miembros del equipo metal (método 2); este método fue eficiente en cuanto a la buena acogida de las personas contactadas pero fue complicada para la adecuación con las zonas de encuesta en Bogotá y para la representatividad sociocultural, aunque se intentó ser lo más diverso en los perfiles. También, este segundo método fue complementado con contactos entregados por las personas entrevistadas a partir de la muestra en Bogotá.

Para el método 1 -basado en la relación con ego- el material fue elaborado por Andrea Salas desde Bogotá, quien recopiló la lista de personas detectadas a través de la encuesta cuantitativa. Igualmente, se revisaron algunos formularios donde aparecía más de un contacto. Finalmente el número de contactos referenciados en la encuesta en Bogotá tanto para Barcelona como en Paris fueron 27 y 15 respectivamente.

La tasa de respuesta fue muy baja: de hecho 14 personas no fueron localizadas de ninguna manera (porque no quisieron o no pudieron darnos contacto). A través de correo electrónico se buscó contacto con nueve personas, después de tres intentos, sólo tres de ellas respondieron afirmativamente para la entrevista. En la segunda misión, tres personas de quienes hemos tenido sus números de teléfono nos han respondido positivamente: una sola ha sido objeto de entrevista al final, la segunda nos dejó dos veces esperando, y la tercera no correspondía a los propósitos de la investigación (una boliviana que nunca había vivido en Bogotá).

El método bola de nieve sólo se utilizó cuando los referidos en la encuesta de Bogotá se iban agotando en cada misión. En ese sentido, se utilizaron dos técnicas para lograr las entrevistas. Una, a partir de los contactos entregados por el equipo METAL y dos, a partir de los contactos entregados por las personas que se iban entrevistando o conocidos en las dos ciudades.

En concreto, cada una de las personas que nosotros conocíamos nos entregaba una lista de colombianos de su conocimiento en cada una de las dos ciudades, y las hemos seleccionado en función de su lugar de residencia antes de la salida, que debía ser dentro o bastante cerca de una de las zonas de encuesta. Muchas entrevistas se iban logrando al final de cada una de las misiones, en la medida en que se iba conociendo la red de bogotanos residentes en cada una de las ciudades. El promedio fue de una entrevista por día.

La principal dificultad fue encontrar a las personas referidas en la encuesta de Bogotá. Varias veces encontramos falta de voluntad por parte de los familiares o amigos de los bogotanos encuestados para que nos entregaran la información completa de los contactos en las dos ciudades. En otras ocasiones, si bien tenían familiares o amigos ya habían perdido el rastro de los mismos. Sólo en los casos en que "ego" tuvo mucho interés en ayudarnos, logramos entrar en relación con los contactos en Barcelona y París. 
Otra dificultad que se presentó en las dos ciudades, con mayor intensidad en Barcelona, es que tres de los referidos ya no vivían en esta ciudad y se habían movido hacia otras dentro del Estado español u otros destinos internacionales. En otros casos se presentó rechazo por desconfianza (un caso en Paris y tres en Barcelona), aparentemente por una situación de ilegalidad o por prevención para entregar la información desde Bogotá. Por otro lado, también puede traducir una mala relación familiar que provoca rechazo de cara a las conexiones que puedan establecer los entrevistadores.

Cabe mencionar la difícil preparación del trabajo de campo en el caso del método 2. Tomar cita desde Colombia no les parecía realista a las personas en Europa, y por otra parte, las estancias en Barcelona y París eran en tiempo limitado. Si se hacían contactos con Bogotá, las condiciones en Barcelona podían haber cambiado. El trabajo se realizaba más fácil en el mismo lugar de encuesta, y así empezaban a surgir un número importante de contactos; sin embargo, era demasiado tarde y no los pudimos valorar.

Luego de la elaboración de la historia de vida en Bogotá y Europa, inmediatamente después se completaban fichas de identificación con el propósito de guardar los detalles de la entrevista y no correr el riesgo del olvido del entrevistador. También este instrumento procuraba recuperar los aspectos más relevantes que posiblemente no estaban en el relato y que podían perderse en el momento de la transcripción; particularidades como el momento de la visita, la descripción del lugar, la actitud del entrevistado, el ambiente, los aspectos relevantes que iban surgiendo, etc. Es decir, una mirada "en caliente de la entrevista". En los casos en que era posible se acompañaba la ficha de fotografías de la vivienda del entrevistado, el entorno y del proceso de entrevista.

A continuación vendría el trabajo de transcripción integral de todas las entrevistas, donde quedaban incluidas todas las expresiones, muletillas, pausas, sentimientos (llanto, risa, sorpresa), etc. Este ejercicio tomó bastante tiempo de la investigación y contó con el apoyo de Naik Miret para algunas de las entrevistas en Europa y estudiantes de pregrado de la Universidad Externado de Colombia para algunas de las entrevistas en Bogotá. La publicación de más de 65 horas de documentos de audio, no aparecen en este texto. Hacen parte del universo analítico que permitió establecer la relación de los objetivos que se plantearon desde un inicio. Algunos apartes de los testimonios se transcriben para reconstruir las atmósferas o para ilustrar mejor en el lector cada una de las partes de los ciclos de vida de las persona que participaron en este trabajo, y desde luego, argumentar sobre los hallazgos de la investigación.

Posteriormente, con base en las transcripciones y las fichas de identificación, el análisis continuó, para el caso de Bogotá con la elaboración de fichas descriptivas y temáticas para cada una de las entrevistas. "En la ficha descriptiva [...] se trata de hacer un resumen de la historia de vida, de dar los principales elementos sobre la vivienda (a partir del cuestionario también), así como incorporar las fotos de la vivienda y del mapa mental. En la ficha temática [...] se trata de colocar las partes de las entrevistas más pertinentes ordenadas con respecto a cada tema de la guía de entrevista acompañada de algunos comentarios." (Dureau, et al., 2011, p. 56). Para las entrevistas en Europa sólo fue elaborada la ficha temática, tomando como modelo la ficha de Bogotá pero adecuándola al énfasis sobre el proceso migratorio que se hacía en Barcelona y Paris.

Finalmente, las entrevistas tuvieron una duración media de una hora, pero algunas fueron muy cortas (tres duraron menos de 40 minutos en Barcelona) o demasiado largas (4 de más de 1 hora y media). Por esta razón, las transcripciones constituyen documentos hasta 60 páginas, complejos para su análisis. 
El uso de la ficha de análisis temática permitió iniciar el proceso de análisis de ese gran volumen de información.

\section{Conclusiones}

El método con varias entradas permitió tener información cuantitativa sobre la movilidad residencial de los individuos así como diversas características asociadas a los hogares, donde aparecía la migración internacional en el ciclo de vida, y por el otro. La elaboración de las historias de vida en tanto información cualitativa consideraba las motivaciones para el cambio residencial en distintas escalas, las prácticas y las representaciones urbanas ya sea en la ciudad de Bogotá o en las dos ciudades europeas. En ese sentido, la información cuantitativa y cualitativa se funde en este tema de manera que permitan comprender con mejor claridad el rol que tiene la movilidad internacional en las transformaciones urbanas en Bogotá.

Este artículo es un aporte en términos de la metodología de la investigación ya que permite reflexionar sobre algunas particularidades de la elaboración de las historias de vida. Sin duda un aspecto de la experiencia individual que contribuye, así sea de manera puntual, a los estudios sociales y de la movilidad espacial.

\section{Referencias Bibliográficas}

Ajuntament de Barcelona. (2011). La població estrangera a Barcelona. Gener 2011. Barcelona: Ajuntament de Barcelona. $166 \mathrm{p}$.

Andrews, G., Kearns, R., Kontos, P., y Wilson, V. (2006). 'Their finest hour': older people, oral histories, and the historical geography of social life. Social \& cultural geography, 7, 153-177.

Bertaux, D. (1980). L'approche biographique: sa validité méthodologique, ses potentialités. Cahiers Internationaux de
Sociologie, LXIX, 197-225.

Bonvalet, C., y Brun, J. (2002). Etat des lieux des recherches sur la mobilité résidentielle en France. In J.-P. Lévy y F. Dureau (Eds.), L'accès à la ville. Les mobilités spatiales en questions (pp. 1564). Paris: L'Harmattan.

Cailly, L. (2007). Capital spatial, stratégies résidentielles et processus d'individualisation. Annales de géographie, 2, 167-187.

Castro, F. (2004). Historial oral: historias de vida e historias barriales. Bogotá: Colectivo de Historia Oral. IED Manuelita Sáenz. Aspectos Siglo XXI. 206 p.

DANE. (2008). Censo general 2005. Nivel Nacional. Bogotá: Departamento Administrativo Nacional de Estadistica. 498 p.

DANE. (s/f). Aproximación a la migración internacional en Colombia a partir del Censo General 2005. Bogotá: DANE. 42 p.

Dureau, F., Córdoba, H., Flórez, C. E., Le Roux, G., Lulle, T., y Miret, N. (2011). Encuestas movilidad espacial Bogotá 2009. Metodología de las encuestas. Documentos CEDE, 23(Número. 63. p. http://www.economia.uniandes.edu. co/publicaciones/dcede2011-23.pdf

Dureau, F., Flórez, C. E., y Hoyos, M. C. (1994, Septiembre). Las formas de movilidad de la población de Bogotá y su impacto sobre la dinámica del área metropolitana: metodología de un sistema de encuestas. Desarrollo y sociedad, $N^{\circ} 34,73-94$.

Grafmeyer, Y. (2004 [1995]). Sociologie urbaine. Paris: Nathan. 128 p.

Khoudour-Castéras, D. (2007). ¿Por qué emigran los colombianos? Un análisis departamental basado en el censo de 2005. Revista de economía institucional, 9, 255-271.

Lelièvre, E. (1999). Collecter des donées des mobilité : des histoires migratoires aux biographies d'entourage. Espace, Populations, Sociétés, $n^{0}$ 2, 195-205.

Londoño, R. (1998). Biografía e historia social: 
el caso de Juan de la Cruz Varela y la Provincia del Sumapaz. In T. Lulle, P. Vargas y L. Zamudio (Eds.), Los usos de la historia de vida en las ciencias sociales I (pp. 19-42). Barcelona: Anthropos. CIDS. IFEA.

Mejía, W., Ortiz, D., Puerta, C., Mena, J., y Díaz, M. (2009). Encuesta nacional 20082009. Resultados generales de migraciones internacionales y remesas. Bogotá: Observatorio Distrital de Migraciones - Observatorio Colombiano de Migraciones. $80 \mathrm{p}$.

Melo, V. (2001). La calle. Espacio geográfico y vivencia urbana en Santa Fe de Bogotá.
Bogotá: Alcaldía Mayor de Bogotá. $111 \mathrm{p}$.

Ramos, L., y Romero, M. (2006). Historia oral y psicología Historia oral: Ensayos y aportes de investgación (Segunda ed., pp. 21-37). México: CIESAS.

Riley, M., y Harvey, D. (2007, June). Oral histories, farm practice and uncovering meaning in the countryside. Social $\mathcal{E}$ cultural geography, 8, No 3, 391-415.

Vega, R., y Castaño, R. (Eds.). (1997). ¡Déjenos hablar! Bogotá: Universidad Pedagógica Nacional. Instituto de Investigación Educativa y Desarrollo Pedagógico. 211 p. 